\title{
Knotty invariants: structure and evolution of magnetized fluids
}

\author{
Ilan Roth \\ Space Sciences, University of California, Berkeley, CA 94720, USA
}

\begin{abstract}
It is conjectured that magnetic configurations may be characterized by the embedded invariants which are extracted from their topological textures. Hence, experimentally observed magnetic structures can be classified by the invariants of the elementary building blocks of the 3$\mathrm{D}$ structures - prime knots. These unique invariants may describe the magnetic configurations in ion drop-outs in solar impulsive events, in photospheric flux ropes carried into the interplanetary medium by the solar wind, and can affect the decay rates for complex magnetic configurations.
\end{abstract}

\section{Introduction}

Evolution of magnetic field is characterized by dynamic changes over broad range of scales. Magnetized fluids are subjected to large-scale injection, turbulent cascade and condensation of magnetic structures at small scales (e.g. Matthaeus, 2008), while observation in the Galaxy (clusters) and in the solar corona indicate dynamo formation of large scale magnetic field over times much shorter than the resistive time-scale. Magnetic helicity, which is conserved over the resistive times has a topological interpretation as a linkage between two not-twisted isolated flux tubes, while its generalization includes both twist and writhe - self-linking number in 2D planar projections which preserve the over/under crossing of the 3D curve. The twist of magnetic field lines at non-linked structures can be seen in interplanetary flux tube of Magnetic Cloud or in magnetospheric flux transfer events. The simplest structure of a closed magnetic field can be interpreted as a two-dimensional manifold, where the magnetic field can be traced as a deformed loop without self-intersections. However, cascade and dynamo result in cutting and pasting of some field lines, when segments of the field may get reconfigured and the new fragments form in the three-dimensional domain more involved structures which cannot be stretched or bent into two-dimensional manifold. The resulting magnetic structures can be depicted as two or more linked loops (interlinked toroidal and poloidal solar or stellar field; Borromean ring) and/or as a non-intersecting magnetic field with a writhe.

Closed field lines in MHD fluid and mathematical knots can be visualized as a collection of entangled, closed loops of a non-self-intersecting curve, continuously deformed in $R^{3}$, following smooth streching and bending in the surrounding viscous fluid. Knots are visualized by $2 \mathrm{D}$ planar projections which preserve the over/under crossing of the $3 \mathrm{D}$ curve. General deformations which allow to manipulate these projections without changing the knot structure were described in the three link moves $R_{j}, \mathrm{j}=1,3$ (Reidemeister, 1926). A knot, which cannot be decomposed into two knots unless one of them is an unknot (equivalent to a loop, Fig. 1) belongs to prime knots, which are the elementary building blocks of the 3-D structures (Fig. 1). Topological knot classification is obtained by assigning to each of its crossings in Fig. 1 a specific algebraic operation connecting the under and over segments of crossings; an invariant is obtained by following self-consistenly the crossings over the whole knot, which results in a unique numerical value or algebraic function which does not change when the knot undergoes the $R_{j}$ deformations. 


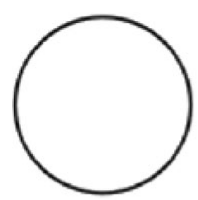

Unknot

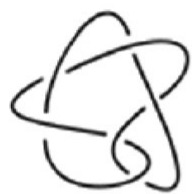

6

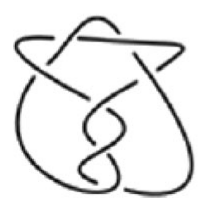

73
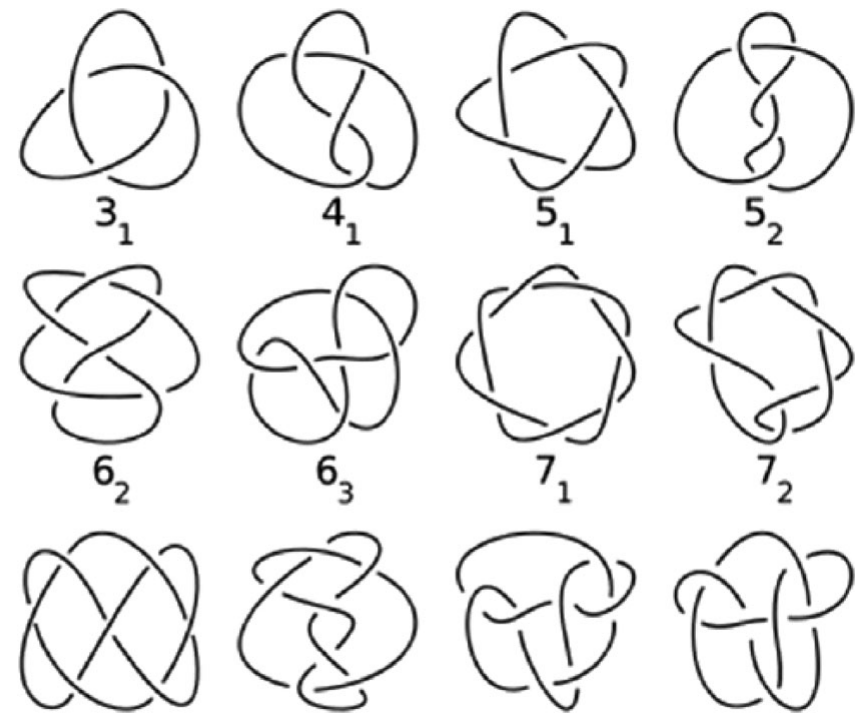

76

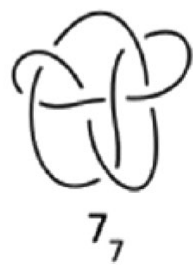

Figure 1. Prime knots.

\section{Implications}

An important invariant is obtained when each under-crossing $\operatorname{arcs} a, b$ and overcrossing $\operatorname{arc} \mathrm{c}$ are classified by the algebraic rule: $a * b=c(t)=t a+(1-t) b$, for variable t. Topological structure is then translated into algebra and labeling the whole knot forms a consistency matrix whose determinant results in a Polynomial $\mathrm{P}(\mathrm{t})$, invariant under $R_{j}$ (Alexander, 1926). Torus-knots, a subset of the prime knots, acquire the following invariants for the Trefoil $3_{1}$, Cinquetfoil $5_{1}$ and Septoil $7_{1}$ (Fig. 1), respectively: $P(t)=t-1+t^{-1}, t^{2}-t+1-t^{-1}+t^{-2}, t^{3}-t^{2}+t-1+t^{-1}-t^{-2}+t-3$.

Solar dynamo process creates magnetic field which emerges from the photosphere as an unknot, with a subset of events in the form of prime knots. These structures, carried by the solar wind without topological modifications because of the extremely low diffusivity, may explain the observed large scale magnetized plasma parcels with distinct orientation, being convected passively from the Sun into 1AU (Borovsky, 2008). Similar formations can be assigned to the drop-out of energetic ions emanating from a compact flare source (Mazur et al. 2000) and applied to the assessment of the decay rates of complex magnetic configurations.

Acknowledgments. This research was supported by NASA grant NNX09AE41G$1 / 14$.

\section{References}

Alexander, J. W., 1928, Trans. Amer. Math. Society, 30, 2758.

Borovsky, J., 2008, Jour. Geoph. Res., 113, A08110

Matthaeus, W. H. et al. 2008, Phys. Rev. Lett., 100, 085003

Mazur, J. et al. , 2000, Astroph. Jour., 532, L79

Reidemeister, K., 1926, Abh. Math. Sem. Univ. Hamburg, 5, 24 